\title{
A comparison of substitution and addition designs for the analysis of competitive interactions in Drosophila melanogaster
}

\section{J. Rodrigues de Miranda and Paul Eggleston}

Department of Genetics, University of Liverpool, P.O. Box 147, Liverpool L69 3BX, U.K.

Two distinct experimental designs are commonly employed for the analysis of competitive interactions in genetically heterogeneous mixtures. These are referred to as substitution and addition designs respectively, and either may be used quantitatively to separate the effects of intra- and inter-genotypic competition. The results presented in this paper relate to two characters which measure competitive success in mixed cultures of Drosophila melanogaster, namely the proportion of developing individuals which survive to emerge as adults and the mean weight of those adults. For each of these characters the estimates of intra- and inter-genotypic competitive values obtained from substitution and addition design experiments were equivalent. The implications of this result with respect to the choice of a suitable experimental design and its application to competition in various plant and animal systems are discussed.

\section{INTRODUCTION}

The importance of competition as an environmental and selective force has long been appreciated (Andrewartha and Birch, 1954; Bakker, 1961). However, detailed investigation of the nature of competitive interactions has been hampered by elusive definitions and the absence of a suitable form of analysis. Recent developments in the analysis of data from competition experiments include the introduction of nearest neighbour and spacing models (Kempton, 1982; Cannel et al., 1984; Benjamin, 1982) which are used primarily in agriculture and forestry related experiments and descriptive, density related regression models (Mather and Caligari, 1981; Spitters 1983a), where the competitive success of a genotype is related to the densities of the various competitors. This latter approach has also been found suitable for the analysis of density related forms of competition in animal systems, as exemplified by the investigation of competition between larvae of Drosophila melanogaster for a constant and limiting amount of food (Mather and Caligari, 1981, 1983; Eggleston, 1985). Mather and Caligari (1981) described two different experimental designs (addition and substitution) for quantitatively separating the effects of intra- and inter-genotypic competition in mixed cultures involving two genotypes. In both cases the fate of a primary, or "indicator" genotype is followed through two types of density series. The first type (monocultures) concerns only the indicator genotype and therefore measures only intra-genotypic competition. The second type (duocultures) mixes indicator competitors with competitors of a secondary, or "associate" genotype and therefore includes both intra- and inter-genotypic competitive effects.

The substitution or replacement design (fig. 1a) has been used extensively since its introduction by de Wit in 1960, albeit without reference to a monoculture density series. This limitation was remedied in largely similar ways by both Mather and Caligari (1981) and Spitters (1983a). In this design, starting from a standard reference density of indicator genotype competitors, individuals are either progressively removed and replaced by associate competitors (forming the duoculture series) or individuals are removed but not replaced (creating the monoculture series). With respect to the indicator genotype only, this creates two similar density series, one with and one without associate genotype competitors. In both cases the performance of the indicator genotype (the dependent variable) is regressed onto the number of indicator genotype competitors removed from the culture. A characteristic, therefore, of the substitution design is that the independent variable is negative. 
The slope obtained from the monoculture regression $\left(b_{m}\right)$ again measures intra-genotypic competition. The slope of the duoculture regression $\left(b_{d}\right)$ measures both intra-genotypic competition among the indicator genotype competitors and the inter-genotypic competitive effect that the associate complement has on the performance of the indicator genotype. If the associate has no effect, then $b_{d}$ would be the same as $b_{m}$. The inter-genotypic competitive value is therefore estimated by the difference between the two slopes $\left(b_{d}-b_{m}\right)$. The intra-genotypic competitive value is quantified similarly as $\left(b_{0}-b_{m}\right), b_{0}$ being the slope obtained if the indicator competitors had not been substituted. One of the useful features of this design is that with the inclusion of a monoculture density series for the secondary genotype the roles of "indicator" and "associate" genotypes can be reversed without having to raise any further duocultures.
In the addition design (fig. $1(b)$ ), starting from a standard reference density of indicator competitors, either more indicator competitors are added, creating the monoculture series, or a comparable number of associate genotype competitors are added, creating the duoculture series. In both cases some measure of the competitive success of the indicator genotype (the dependent variable) is regressed onto culture density (the independent variable).

In the case of the monoculture series, the number of additional indicator genotype competitors forms the independent variable, whereas in the duoculture series this role is performed by the number of additional associate genotype competitors. The slope of the monoculture regression $\left(b_{m}\right)$ is representative of the magnitude of intragenotypic competition and in the duoculture regression the slope $\left(b_{d}\right)$ measures the effect that the associate competitors have on the competitive
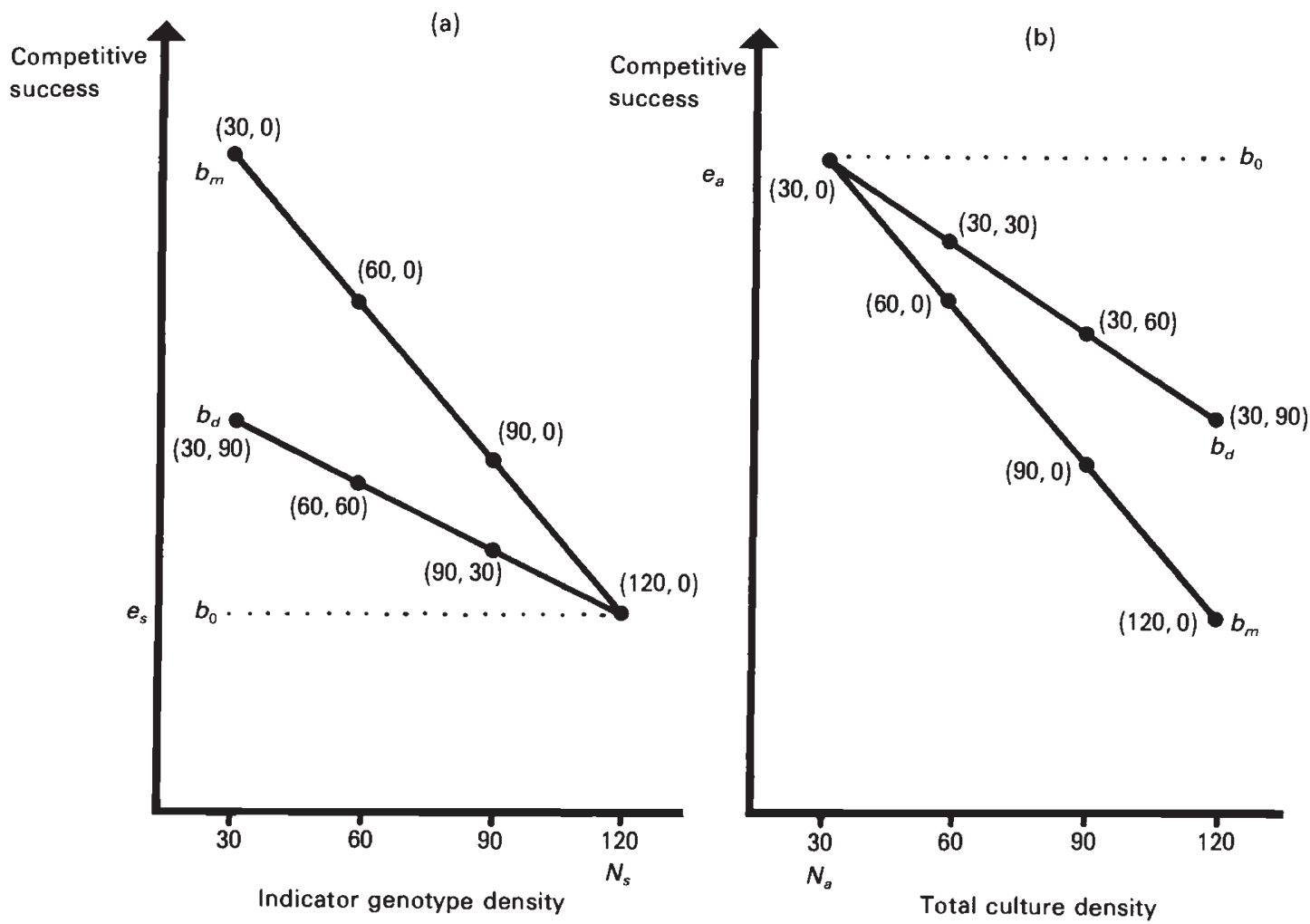

Figure 1 Comparisons of the substitution (a) and addition (b) designs for the analysis of competitive interactions. $N_{s}$ and $N_{a}$ refer to the reference densities for the substitution and addition designs respectively, with $e_{s}$ and $e_{a}$ the indicator genotype performance at these reference densities. $b_{m}$ represents the slope of the regression when the indicator genotype is raised in monoculture and $b_{d}$ represents a similar slope when the indicator is raised in duoculture with an associate genotype. The figures in brackets indicate the density of indicator and associate genotype competitors respectively. The intra-genotypic competitive value $c_{x x}$ is found as $\left(b_{0}-b_{m}\right)$ for each design. The inter-genotypic competitive value $c_{x y}$ is found as $\left(b_{d}-b_{m}\right)$ for the substitution design and as $\left(b_{0}-b_{d}\right)$ for the addition design. 
success of the indicator genotype. Since the number of indicator competitors remains constant throughout the duoculture density series, the amount of intra-genotypic competition remains constant and any change from the norm must be due to the inter-genotypic competition exerted by the associate genotype upon the indicator. These computations relate only to the effects on the indicator genotype. In order to determine the effects of competition on the associate genotype a similar experiment has to be conducted in which the roles of "indicator" and "associate" are reversed. The estimates for intra- and intergenotypic competition are generally referred to as competition or $c$-values where, for example, $c_{x y}$ represents the effect of associate genotype $Y$ on the performance of indicator genotype $X$.

Although competition per se cannot be assumed to differ between the two designs, the choice of either a substitution or addition design may still be important. For example, Spitters and van den Bergh (1982) indicated that the invasion of a crop (seeded at uniform density) by weeds (at variable density) clearly conforms more to an addition design, whereas intercropping (the mixing of different crops or cultivars such that the overall density or density equivalent remains constant), relates more to a substitution design. In this paper we show that, using the analysis developed by Mather and Caligari (1981) and the Drosophila melanogaster model system, the competitive values for intra- and inter-genotypic competition obtained in a substitution design are equivalent to those obtained with an addition design. The general implications of these results with respect to the choice of a suitable competition analysis are discussed.

\section{EXPERIMENTAL DETAILS}

The procedures used were essentially the same as those employed by Eggleston (1985). Four wild type inbred strains (T5, T19, T25, and T27) derived from the Texas population of Drosophila melanogaster (Linney et al., 1971) were investigated. Two further strains, derived from T19 and T25 and identical apart from the inclusion of a yellow body colour marker were also involved. These were designated $\mathrm{y}^{2} \mathrm{~T} 19$ and $\mathrm{y}^{2} \mathrm{~T} 25$. The introduction of the $y^{2}$ allele has a negligible effect on the competitive behaviour of the Texas strains (Caligari, 1980; Mather and Caligari, 1983) and makes it possible to distinguish the indicator and associate competitors in the duocultures. Competi- tion took place in $2.5 \mathrm{~cm} \times 7.5 \mathrm{~cm}$ glass vials, each containing $5.0 \mathrm{ml} 2$ per cent Bacto agar to which $15 \mathrm{ml} / 1$ of 10 per cent Nipagin in ethanol was added as an antifungal agent. $45 \mathrm{mg}$ live yeast (YSC-2, Sigma) dispensed as a $100 \mathrm{mg} / \mathrm{ml}$ solution in distilled water, was deposited on the surface of the agar in each vial. The vials were dried overnight at $25^{\circ} \mathrm{C}$ and stored at $4^{\circ} \mathrm{C}$ until use. Competition was started by seeding the competition vials with the required number of eggs from the various genotypes. Eggs were collected during a short ( $2 \mathrm{~h} 30 \mathrm{~min}$ ) laying period in order to minimise developmental heterogeneity. For each of the four wild type and two yellow strains one monoculture density series was raised, consisting of $30,60,90$, and 120 eggs per culture. For every possible combination of wild type and yellow strains, the following duocultures were raised.

$\begin{array}{lllllll}\text { wild type: } & 30 & 30 & 30 & 60 & 60 & 90 \\ \text { yellow type: } & 30 & 60 & 90 & 30 & 60 & 30 \\ & \text { AB } & \text { A } & \text { AS } & \text { B } & \text { S } & \text { BS }\end{array}$

Those cultures marked A or B form part of the addition design, taking either the wild type strain as the indicator genotype (cultures A) or the yellow strain as the indicator genotype (cultures B). The cultures marked $\mathrm{S}$ form part of the substitution design, where either the wild type or the yellow strain can be taken as the indicator genotype.

To validate the assumption that a uniformly high proportion of the eggs of each genotype hatched into competing larvae, samples of 50 eggs were taken from each strain for each replicate experiment in order to determine the egg hatchabilities. These hatchabilities were relatively uniform at approximately 80 per cent in all cases.

The entire experiment, comprising all of the necessary substitution and addition cultures, was raised in duplicate on two separate occasions, providing essentially four replicates. All cultures within each replicate were individually randomised and all four replicates were raised at $25 \pm 0 \cdot 5^{\circ} \mathrm{C}$. Since there was no significant difference between using the means of the two occasions or all four replicates as the source of the error variation, the latter option was used.

Any analysis of competition must include one or more characters that measure competitive success. The two characters used in this investigation, where food is the limiting resource, were the proportion of seeded eggs surviving to adulthood $(p)$ and the mean weight of those adults $(\bar{w})$. For 10 days following the emergence of the first adults, flies were collected daily and separated by body colour (yellow or wild type) and sex, recording 
the number and weight of each group before discarding them. After calculating $p$ and $\bar{w}$ these variables were transformed so as to approach a linear relationship with density. This was regarded as being preferable to the use of untransformed data in higher order regressions (Mather and Caligari, 1981; Spitters, 1983a; Spitters et al, 1986). Consequently, $p$ was transformed to $p_{a}$ using the angular transformation $p_{a}=\sin ^{-1} \sqrt{p}$. The mean weight $\bar{w}$ was converted to $1 / \bar{w}$. Bakker (1961) has shown that the mean adult weight in Drosophila is linearly related to the amount of food available per larva. Density effectively measures the number of larvae per unit food and is thus, logically, inversely related to the mean adult weight.

\section{ANALYTICAL DETAILS}

In the original design of Mather and Caligari (1981), a single reference density was proposed for both the addition and substitution designs. However, the choice of characters used here made it impossible to use a single reference density and still retain linearity over the range of densities employed. Out of a variety of options we chose to use different reference densities for the addition and substitution designs. One of the consequences of this choice was that the monoculture slopes $\left(b_{m}\right)$ for the addition and substitution designs were derived from the same set of cultures (fig. 1). In such a case (as, in fact, in Mather and Calgari's original design), a single estimate for $b_{m}$ should apply to both designs. If, however, the substitution and addition experiments are analysed independently, two estimates for $b_{m}$ (though very similar) are obtained. For this reason a combined analysis was used where the two designs contributed in equal parts to the estimation of $b_{m}$. This yielded the following set of least squares equations;

\section{Substitution \\ $Q_{m s}=\left(Y_{m}-e_{s}-b_{m} X_{m s}\right)^{2}$ \\ $Q_{d s 1}=\left(Y_{d s 1}-e_{s}-b_{d s 1} X_{d s 1}\right)^{2}$ \\ $Q_{d s 2}=\left(Y_{d s 2}-e_{s}-b_{d s 2} X_{d s 2}\right)^{2}$}

etc.
Addition

$$
\begin{aligned}
& Q_{m a}=\left(Y_{m}-e_{a}-b_{m} X_{m a}\right)^{2} \\
& Q_{d a 1}=\left(Y_{d a 1}-e_{a}-b_{d a 1} X_{d a 1}\right)^{2} \\
& Q_{d a 2}=\left(Y_{d a 2}-e_{a}-b_{d a 2} X_{d a 2}\right)^{2}
\end{aligned}
$$

etc. where $Q_{m s}$ and $Q_{m a}$ represent the monoculture regressions for the substitution and addition designs respectively, while $Q_{d s}$ and $Q_{d a}$ similarly represent a series of duoculture regressions. $Y_{m}$, $Y_{d s}$ and $Y_{d a}$ are the observed values (means over the four replicates) for the indicator performance in monoculture and in the substitution and the addition duocultures respectively. $X_{m s}$ and $X_{d s}$ represent the deviation of the indicator genotype density from the substitution reference density $\left(X-N_{s}\right)$ for the monocultures and the duocultures respectively. Similarly, $X_{m a}$ and $X_{d a}$ represent the deviation of the total culture density from the addition reference density $\left(X-N_{a}\right)$ for the monocultures and the duocultures respectively. $e_{s}$, $e_{a}, b_{m}, b_{d s}$ and $b_{d a}$ are the estimated parameters defined previously (fig. 1). These equations were combined as follows to obtain a single regression quantity;

$$
\begin{aligned}
Q= & \left(Q_{m s}+Q_{m a}\right) / 2+Q_{d s 1}+Q_{d s 2}+\cdots \\
& +Q_{d a 1}+Q_{d a 2}+\cdots
\end{aligned}
$$

Minimising $Q$ with respect to each of the estimated parameters in turn gave the matrix of normal equations shown at the head of the following page. Utilising Mather and Calgari's terminology, $S$ represents summation, $n_{s}$ is the number of substitution duoculture observations plus half the number of monoculture observations and $n_{a}$ is the number of addition duoculture observations plus half the monoculture observations. With a single reference density, $n_{s}$ and $n_{a}$ would be combined into a single value, $n$, representing the total number of observations in the analysis. Certain other definitions $\left[\boldsymbol{S}\left(\boldsymbol{X}_{m s}\right), \boldsymbol{S}\left(\boldsymbol{X}_{m a}\right)\right.$ and $\left.\boldsymbol{S}\left(Y_{d s}\right), \boldsymbol{S}\left(Y_{d a}\right)\right]$ would also be combined into a single quantity. In such a case, the generalised matrix suggested by Mather and Caligari (1981) should be used. The $J$ matrix is inverted and the expression $\hat{\boldsymbol{M}}=\boldsymbol{J}^{-1} . \boldsymbol{S}$ used to derive estimates of each parameter.

In the few instances that no flies of a particular genotype emerged from a culture, Bartlett's (1947) empirical correction of $0+1 /(4 N)$ where $N$ is the number of seeded eggs, was used to replace the missing value for $p . p_{a}$ was then calculated as before. For $1 / \bar{w}$, the mean value of the remaining replicates was used to substitute for the missing replicate observation. Since these corrections will tend to reduce the error variance, one degree of freedom was lost for each correction. Throughout the whole experiment, no more that ten entries were lost, out of 480 , and no plot in the regression was based on less than two replicates. The basic error variances were calculated separately for each indicator genotype from the differences between the four replicate observations. These error variances were heterogenous, and all the subsequent analyses therefore utilised the error variance appropriate to the indicator genotype under consideration. The adequacy of the linear regression model for describing competition was tested by calculating the residual variation around the 
$\left[\begin{array}{c}\frac{1}{2} S\left(Y_{m}\right)+S\left(Y_{d s}\right) \\ \frac{1}{2} S\left(Y_{m}\right)+S\left(Y_{d a}\right) \\ \frac{1}{2} S\left(Y_{m}\left(X_{m s}+X_{m a}\right)\right) \\ S\left(Y_{d s 1} X_{d s 1}\right) \\ S\left(Y_{d s 2} X_{d s 2}\right) \\ S\left(Y_{d a 1} X_{d a 1}\right) \\ S\left(Y_{d a 2} X_{d a 2}\right)\end{array}\right]\left[\begin{array}{c}n_{s} \\ 0 \\ \frac{1}{2} S\left(X_{m s}\right) \\ S\left(X_{d s 1}\right) \\ S\left(X_{d s 2}\right) \\ 0 \\ 0\end{array}\right.$

$S$

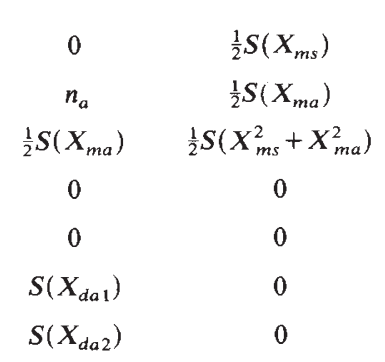

$S\left(X_{d a 2}\right)$

$S\left(X_{d s 1}\right)$
0
0
$S\left(X_{d s 1}^{2}\right)$
0
0
0

$\boldsymbol{J}$

$S\left(X_{d s 2}\right)$
0
0
0
$S\left(X_{d s 2}^{2}\right)$
0
0

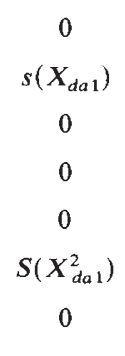

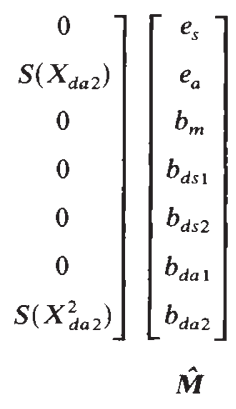

regression lines, which should not exceed one quarter of the appropriate error variance (since each plot in the regression was a mean over four replicate observations). In all cases, the linear model was found to be adequate. The error variances associated with each of the estimated parameters in $\hat{\boldsymbol{M}}$ were obtained by multiplying the corresponding entry in the leading diagonal of the $\boldsymbol{J}^{-1}$ matrix with one quarter of the replicate error variance. The error variances associated with the $c$-values are the same as those for the slopes from which the $c$-values were derived, except where $c_{x y}=\left(b_{d s}-b_{m}\right)$. In this case we have two estimated slopes, each with their error variance and a covariance (represented by the off-diagonal terms in the $\boldsymbol{J}^{-1}$ matrix). The covariance terms were therefore subtracted from the combined error variance in a manner similar to that described by Mather and Caligari (1981).

\section{RESULTS}

The intra- and inter-genotypic competitive values obtained throughout the experiment are given in table 1 , and part of this information is depicted graphically in fig. 2. Several general observations can be inferred from these data, the most striking of which is the variability of competitive behaviour, even within such a small sample of genotypes. This concurs with the large amount of variation in competitive ability described by Eggleston (1985). Secondly, the competitive values obtained in the addition and substitution designs are clearly very similar. Thirdly, there is only moderate correlation between the competitive values obtained for $p_{a}$ and $1 / \bar{w}(r=0.551)$ suggesting that the competitive determinants for survival are to a large extent different from those which determine mean weight (or rather, $1 / \bar{w}$ ). This also indicates that the inferred competitive ability depends on the choice of character. A similar situation arises in plant crop yield-density experiments, where either the total biomass or any component of the biomass can be used to measure competitive success, with differing results (Spitters, 1983b).

The main purpose of these experiments was to determine whether the values for intra- and intergenotypic competition ( $c$-values) that may be obtained using the previously untested addition design of Mather and Caligari (1981) are equivalent to those commonly obtained using the more familiar substitution design. These values are shown in table 1 for both $p_{a}$ and $1 / \bar{w}$. The leading diagonal gives the estimates of intra-genotypic competition obtained from each monoculture density series. These estimates are identical for both the substitution and the addition designs (due to the nature of the combined analysis) and are therefore excluded from any analysis testing the differences between the two designs. The remaining figures in the table are estimates of the intergenotypic competition and these are different for the two designs. For the purpose of analysis table 1 can be divided conveniently into two blocks of results; block A, which includes all those results where $y^{2}$ T19 and $y^{2}$ T25 are associate genotype competitors and block $\mathrm{B}$, which includes those results where $\mathrm{y}^{2} \mathrm{~T} 19$ and $\mathrm{y}^{2} \mathrm{~T} 25$ are indicator genotype competitors. This separation of the results into two blocks makes it possible to investigate the variation between indicators and between associates, in addition to any differences between the two designs. Each block, therefore, was analysed independently and a summary of the analyses of variance is given in table 2 . It should be noted that none of the interaction items in the analysis were significant and these have therefore been omitted from table 2. The error variance for each analysis was obtained by pooling the error variances for each of the $c$-values involved. The conclusions that can be drawn from this analysis are clear. There is no significant difference between the two designs with respect to the competitive values obtained for either character. Only the variation between the six genotypes, whether as 
Table 1 Estimates of the competitive values obtained throughout the experiment. The leading diagonal gives the intra-genotypic values obtained in monoculture and the off-diagonal terms the inter-genotypic values obtained in duoculture for various combinations of indicator and associate genotypes. The two upper entries in each cell of the table give the values obtained for the character $p_{a}$ in the addition and substitution designs respectively. The lower two entries give equivalent values obtained for the character $1 / \bar{w}$. For each genotype, the mean aggression (taken over all indicators) and the mean response (taken over all associates) is given with respect to the characters $p_{a}$ (upper figure) and $1 / \bar{w}$ (lower figure). See text for details. All entries with respect to the character $1 / \bar{w}$ have been multiplied by $10^{2}$ for ease of presentation

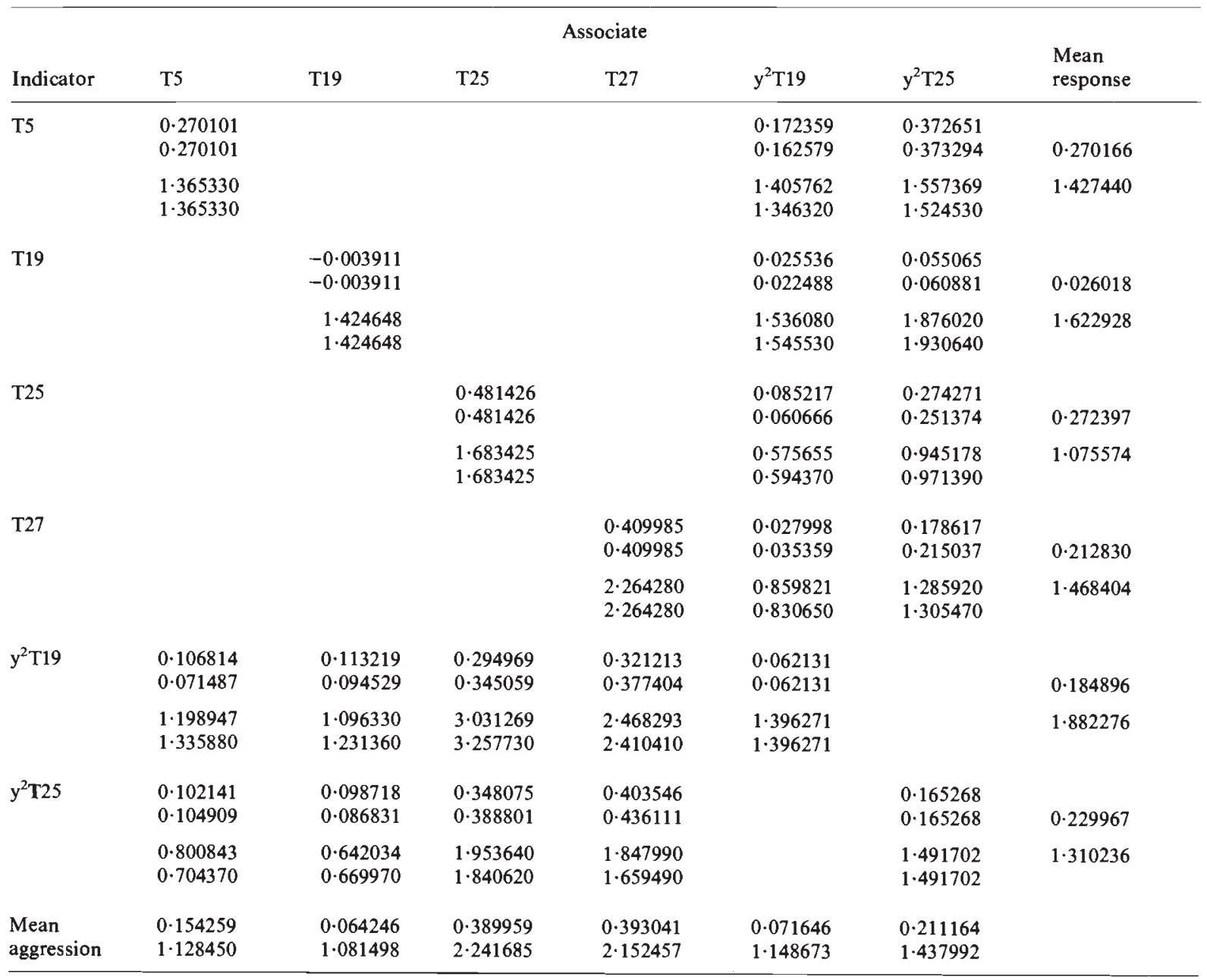

indicators or as associates was found to be significant, with the further observation that there is, generally, more variation between the genotypes as associates than between the same genotypes as indicators. This is in agreement with previous findings (Mather and Caligari, 1983; Eggleston, 1985).

The $c$-values (table 1) represent the competitive behaviour of a range of indicator genotypes in the presence of particular associate genotypes and therefore contain information both about the sensitivity of the indicator genotypes to competition (response) and information about the relative competitive strength of the associate genotypes (aggression). The dissemination of $c$-values into components of aggression and response can proceed using an orthogonal analysis as described by Mather and Caligari (1983) and Eggleston (1985) which investigates the relationships between any two genotypes. This yields a series of independent (and unrelated) estimates for aggression ( $a$ ), response $(r)$ and their interaction $(i)$ which apply exclusively to the pair of genotypes in question. Alternatively, more general estimates of the average aggression (taken over all indicators) and the average response (taken over all associates) can be obtained (Breese and Hill, 1973). These values represent the relative competitive behaviour 

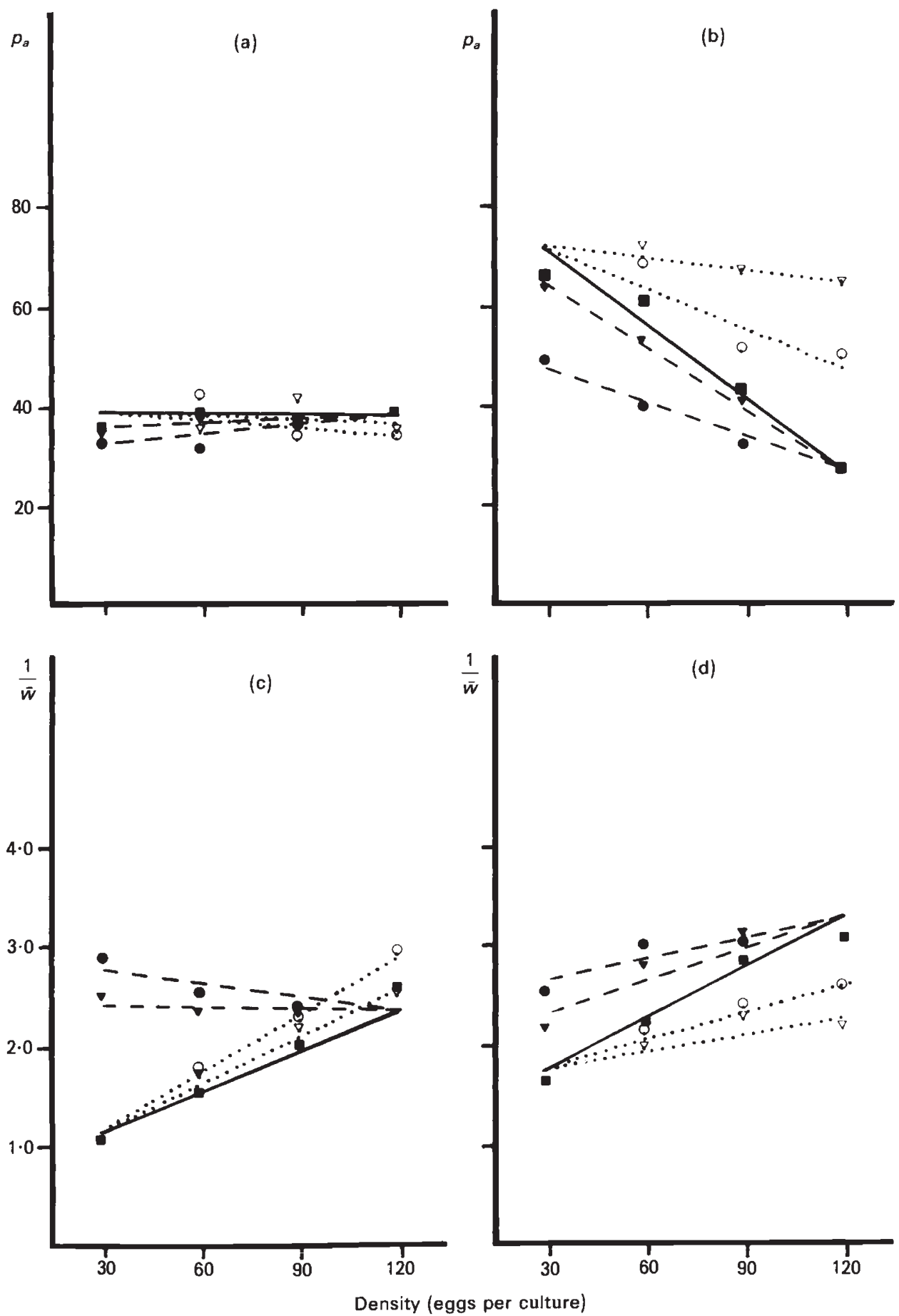

Figure 2 Estimated regression lines relating the characters $p_{a}$ and $1 / \bar{w}$ to culture density for both substitution and addition designs. The indicator genotype in (a) and (c) is T19 whereas in (b) and (d) the indicator genotype is T25. In each case the indicator genotype monoculture is represented by an unbroken line. Substitution and addition design duocultures are represented by broken and dotted lines respectively. The observed points are shown as follows. Solid squares represent the indicator genotype monocultures. Solid and open triangles respectively represent substitution and addition design duocultures with $\mathrm{y}^{2} \mathrm{~T} 19$ as the associate genotype. Solid and open circles respectively represent substitution and addition design duocultures with $\mathrm{y}^{2} \mathrm{~T} 25$ as the associate genotype. The X-axis represents either indicator genotype density (substitution design) or total density (addition design). 
Table 2 A summary of the analysis of variance of the inter-genotypic competitive values given in table 1 for (a) the character $p_{a}$ and (b) the character $1 / \vec{w}$. Block $A$ relates to those values obtained when the genotypes $\mathrm{y}^{2} \mathrm{~T} 19$ and $\mathrm{y}^{2} \mathrm{~T} 25$ are taken to be associate competitors. Block B represents these same genotypes taken as indicator competitors. All leading diagonal values from table 1 have been omitted from this analysis because they are identical in both designs. To assist presentation, all MS for character $p_{a}$ have been multiplied by $10^{3}$ and those for character $1 / \bar{w}$ by $10^{6}$. The levels of significance are given as NS $(P>0.05)$; (a) * $(P=0.05-0.01)$; $^{* *}(P=0.01-0.001) ;{ }^{* * *} P<0.001$

\begin{tabular}{|c|c|c|c|c|c|c|c|c|}
\hline \multirow[b]{2}{*}{ Item } & \multirow[b]{2}{*}{$\mathrm{df}$} & \multicolumn{3}{|c|}{ Block A } & \multicolumn{4}{|c|}{ Block B } \\
\hline & & MS & $\mathrm{F}$ & P & $\mathrm{df}$ & MS & $\mathrm{F}$ & $\mathbf{P}$ \\
\hline Designs & 1 & 0.0062 & 0.003 & NS & 1 & 0.8473 & 0.668 & NS \\
\hline Indicators & 3 & $37 \cdot 2222$ & $17 \cdot 155$ & $* * *$ & 1 & 3.7343 & 2.944 & NS \\
\hline Associates & 1 & 88.4325 & $40 \cdot 715$ & *** & 3 & $96 \cdot 1867$ & 75.834 & *** \\
\hline Error & 192 & $2 \cdot 1698$ & & & 158 & $1 \cdot 2698$ & & \\
\hline
\end{tabular}

(b)

\begin{tabular}{|c|c|c|c|c|c|c|c|c|}
\hline \multirow[b]{2}{*}{ Item } & \multicolumn{4}{|c|}{ Block A } & \multicolumn{4}{|c|}{ Block B } \\
\hline & $\mathrm{df}$ & MS & $\mathrm{F}$ & $\mathrm{P}$ & $\mathrm{df}$ & MS & $F$ & $\mathbf{P}$ \\
\hline Designs & 1 & 0.0001 & 0.0004 & NS & 1 & 0.0339 & 0.008 & NS \\
\hline Associates & 1 & $45 \cdot 6422$ & $19 \cdot 875$ & $* * *$ & 3 & $255 \cdot 2060$ & 63.095 & $* * *$ \\
\hline Error & 192 & $2 \cdot 2965$ & & & 158 & $4 \cdot 0448$ & & \\
\hline
\end{tabular}

of the six genotypes with respect to their potential to exert aggression and to respond to such aggression. The estimates obtained for mean aggression and mean response of the six genotypes are shown in table 1 and are reproduced in rank order together with their standard errors in table 3 . These values were obtained by first pooling the competitive values from the addition and substitution designs and subsequently by summing over the appropriate genotypes. The standard errors associated with these estimates were adjusted to take account of the number of observations contributing to the mean values of aggression and response.

The data in table 3 show that, as observed previously (Eggleston, 1985), there is a significant amount of variation in both aggression and response, with generally more variation in aggression $\left[\chi_{(5)}^{2}=495.48\right.$ and $\chi_{(5)}^{2}=270.07$ for the characters $p_{a}$ and $1 / \bar{w}$ respectively] than in

Table 3 Estimates of the mean aggression and mean response of the six genotypes involved in the experiment for (a) character $p_{a}$ and (b) character $1 / \bar{w}$. All values relating to the character $1 / \bar{w}$ have been multiplied by $10^{2}$ for ease of presentation. Levels of significance for the deviation of each estimate from zero are given as in table 2 (a)

\begin{tabular}{|c|c|c|c|c|c|c|}
\hline Rank & Genotype & Mean aggression & $P$ & Genotype & Mean response & $P$ \\
\hline 1 & $\mathrm{~T} 27$ & $0.393041 \pm 0.016083$ & $* * *$ & $\mathrm{~T} 25$ & $0.272397 \pm 0.019244$ & $* * *$ \\
\hline 2 & $\mathrm{~T} 25$ & $0.389960 \pm 0.015496$ & $* * *$ & T5 & $0 \cdot 270166 \pm 0.019341$ & $* * *$ \\
\hline 4 & T5 & $0.154259 \pm 0.015521$ & $* * *$ & $\mathrm{~T} 27$ & $0.212830 \pm 0.021412$ & $* * *$ \\
\hline 5 & $\mathrm{y}^{2} \mathrm{~T} 19$ & $0.071646 \pm 0.013259$ & $* * *$ & $\mathrm{y}^{2} \mathrm{~T} 19$ & $0.184896 \pm 0.009179$ & $* * *$ \\
\hline 6 & T19 & $0.064246 \pm 0.014122$ & $* * *$ & T19 & $0.026018 \pm 0.013310$ & NS \\
\hline
\end{tabular}

(b)

\begin{tabular}{llllll}
\hline Rank & Genotype & Mean aggression & $P$ & Genotype & Mean response \\
\hline 1 & T25 & $2 \cdot 241685 \pm 0.076199$ & $* * *$ & $\mathrm{y}^{2} \mathrm{~T} 19$ & $1 \cdot 882276 \pm 0 \cdot 069173$ \\
2 & T27 & $2 \cdot 152457 \pm 0.075773$ & $* * *$ & T19 & $1 \cdot 622928 \pm 0 \cdot 059545$ \\
3 & $\mathrm{y}^{2} \mathrm{~T} 25$ & $1 \cdot 438041 \pm 0.048939$ & $* * *$ & T27 & $1 \cdot 468404 \pm 0 \cdot 052081$ \\
4 & $\mathrm{y}^{2}$ T19 & $1 \cdot 148673 \pm 0.052931$ & $* * *$ & T5 & $1 \cdot 427440 \pm 0.072996$ \\
5 & T5 & $1 \cdot 128450 \pm 0.079325$ & $* * *$ & $\mathrm{y}^{2} \mathrm{~T} 25$ & $1 \cdot 310236 \pm 0 \cdot 055508$ \\
6 & T19 & $1 \cdot 081498 \pm 0.076922$ & $* * *$ & T25 & $1 \cdot 075574 \pm 0 \cdot 054950$ \\
\hline
\end{tabular}


response $\left[\chi_{(5)}^{2}=206 \cdot 27\right.$ for $p_{a}$ and $\chi_{(5)}^{2}=101.64$ for $1 / \bar{w}]$. All of these $\chi^{2}$ values are, of course, highly significant. An analysis of the relationship between mean aggression and mean response values revealed non-significant correlation coefficients of $r=0.58$ and $r=-0.65$ for the characters $p_{a}$ and $1 / \bar{w}$ respectively. This independence of aggression and response is in agreement with earlier reports (Eggleston, 1985).

\section{DISCUSSION}

The ascendancy of regression models to describe competitive interactions is primarily due to their popularity with many agricultural experimentalists (Breese and Hill, 1973; Mather and Caligari, 1981; Mather, Hill and Caligari, 1982). Experiments of this type are usually concerned either with the competitive effect of weeds on crop yield and the efficacy of various weed control measures (O'Sullivan et al., 1982; Minjas and Runeckles, 1984; Baan-Hofman and Ennik, 1982; Vernon and Parker, 1983; Spitters and van den Bergh, 1982), or with the use of competitive mixing of different crops or cultivars to stabilise or improve the total yield (Chowdury and Hodgson, 1982; Spitters, 1983b; Salter et al., 1985; Mead and Riley, 1981). These experiments generally differ both in design and analysis with the weed-crop interactions conforming more to an addition type design and the mixed cropping experiments usually being more suited to a substitution design analysis. In this paper we show that, with minor modifications, the experimental procedure of Mather and Caligari (1981) yields equivalent values for intra- and intergenotypic competition in both substitution and addition designs, thereby providing a means to apply the results and conclusions from one design to the other. One of the implications of this result is that in a situation where all four competitive interactions present in a duoculture series (i.e. the intra-genotypic effects of genotypes $\mathrm{X}$ and $\mathrm{Y}$ on their own performance and the inter-genotypic effect of $\mathrm{X}$ on $\mathrm{Y}$ and of $\mathrm{Y}$ on $\mathrm{X}$ ) are to be determined, the substitution design may be a more economical way of obtaining these estimates, even if their final application is more relevant to an addition type design. The reason for this is, of course, that in a substitution design duoculture either of the pair of genotypes may be considered as the indicator. In the addition design, however, a separate duoculture density series must be used for each genotype which is taken to be the indicator. Several queries remain, however, as to the universality of the present design and the possi- bility of applying experimental results. Spitters et al. (1986) discovered an asymptotic relationship in his addition design where the reduction in crop yield per weed plant was less for each extra weed plant added to the crop-weed mixture. This diminishing effectiveness of the added weeds he ascribed to increased intra-genotypic competition between the weeds. In this experiment, however, the linear relationship in the addition design was found to be satisfactory throughout the density range employed, up to an associate-indicator ratio of $3: 1$. The second question regarding the equivalence of substitution and addition derived $c$-values concerns the fact that these results apply only to the particular reference densities involved. Although further analysis of the data suggests that the use of other densities within the range as the addition design reference density does not markedly affect the similarity of the substitution and addition design $c$-values, it is not certain whether the equivalence will extend to any reference density. A similar problem exists with respect to the substitution design where the values for intra- and inter-genotypic competition depend on the choice of the reference density. Spitters and van den Bergh (1982) tried to solve this problem by re-defining density to form a more flexible function involving components for resource access and use, thereby uncoupling the dependence of the estimation of competitive success from the choice of reference density, as well as allowing for dynamic changes in the competitive interactions.

The present study is completed by the estimation of mean aggression and mean response values for the six genotypes. These are extremely variable for both $p_{a}$ and $1 / \bar{w}$, with aggression showing nearly twice the variability of response. Mean aggression and response values show no significant correlation and this supports an earlier suggestion that these characters might be under independent genetic control or at least separately adjustable by selection. For the character $1 / \bar{w}$ the suggestion is that the optimum competitive strategy of high aggression and low response has been fixed in the stronger competitors, whereas the weaker competitors display a low aggression and a high response. Confirmation of these early indications based on a limited range of genotypes, must wait until a larger sample of the available genotypes has been surveyed for this character.

Acknowledgements We are grateful to Professor Sir Kenneth Mather for his helpful discussion and to Professor P. D. S. Caligari for the donation of the yellow marked inbred lines. We acknowledge the financial assistance of the Science and Engineering Research Council. 


\section{REFERENCES}

ANDREWARTHA, H. G. AND BIRCH, L. C. 1954. The distribution and abundance of animals, University of Chicago Press, Chicago.

BAAN-HOFMAN, T. AND ENNIK, G. C. 1982. The effect of root mass of perennial ryegrass (Lolium perenne L.) on the competitive ability with respect to couchgrass (Elytrigia repens (L.) Desv.). Neth. J. Agric. Sci., 30, 275-283.

BAKKER, K. 1961. An analysis of factors which determine success in competition for food among larvae of Drosophila melanogaster. Arch. Neerland. Zool., 14, 200-281.

BARTLETT, M. S. 1947. The use of transformations. Biometrics, 3, 39-53.

BENJAMIN, L. R. 1982. A model to predict the effect of complex row spacings on the yields of root crops. J. Agric. Sci., Cam., 98, 131-139.

BREESE, E. L. AND HILL, J. 1973. Regression analysis of interactions between competing species. Heredity, 31, 181-200.

CALIGARI, P. D. S. 1980. Competitive interactions in Drosophila melanogaster. I. Monocultures. Heredity, 45, 219-231.

CANNELL, M. G. R., ROTHERY, P. AND FORD, E. D. 1984. Competition within stands of Picea stichensis and Pinus contorta. Annals of Botany, 53, 349-362.

CHOWDURY, A. R. AND HODGSON, D. R. 1982. Growth and yield in pure and mixed crops of potato cultivars. J. Agric. Sci., Camb., 98, 505-516.

EGGLESTON, P. 1985. Variation for aggression and response in the competitive interactions of Drosophila melanogaster. Heredity, 54, 43-51.

KEMPTON, R. A. 1982. Adjustment for competition between varieties in plant breeding trials. J. Agric. Sci., Camb., 98, 599-611.

LINNEY, R., BARNES, B. W. AND KEARSEY, M. J. 1971. Variation for metrical characters in Drosophila populations. Heredity, $27,163-174$.

MATHER, K. AND CALIGARI, P. D. S. 1981. Competitive interactions in Drosophila melanogaster II. Measurement of competition. Heredity, 46, 239-254.
MATHER, K. AND CAligari, P. D. S. 1983. Pressure and response in competitive interactions. Heredity, 51, 435-454.

MATHER, K. HILL, J. AND CALIGARI, P. D. S. 1982. Analysis of competitive ability among genotypes of perennial ryegrass. Heredity, 48, 421-434.

MEAD, R. AND RILEY, J. 1981. A review of statistical ideas relevant to intercropping research. J. Royal Stat. Soc. A., 144, 462-509.

MINJAS, A. N. AND RUNECKLES, V. C. 1984. Application of monoculture yield/density relationships to plant competition in binary additive series. Annals of Botany, 53, 599-606.

O'SUllivan, P. A., KoSSATZ, V. C., WEISS, G. M. AND DEW, D. 1982. An approach to estimating yield loss of barley due to Canada thistle. Can. J. Plant. Sci., 62, 725-731.

SALTER, P. J., AKEHURST, J. M. AND MORRIS, G. E. L. 1985. An agronomic and economic study of intercropping brussels sprouts and summer cabbage. Exp. Agric., 21, 153-167.

SPITTERS, C. J. T. $1983 \mathrm{a}$. An alternative approach to the analysis of mixed cropping experiments. 1. Estimation of competitive effects. Neth. J. Agric. Sci., 31, 1-11.

SPITTERS, C. J. T. $1983 \mathrm{~b}$. An alternative approach to the analysis of mixed cropping experiments. 2. Marketable yield. Neth. J. Agric. Sci., 31, 143-155.

SPITTERS, C. J. T. AND VAN DEN BERGH, J. P. 1982. Competition between crop and weeds: A system approach. Holzner, W. and Numata, N. (eds), In Biology and ecology of weeds, Dr. W. Junk Publishers, The Hague, Chapt. 12, 137-148.

SPITTERS, C. J. T., KROPF, M. J. AND DE GROOT, W. 1986. Use of the hyperbolic yield-density equation to describe cropweed competition. (Submitted Annals Applied Biology)

VERNON, R. AND PARKER, J. M. H. 1983. Maize-weed competition: implications for tropical small-farm weed control research. Expl. Agric., 19, 341-347.

DE WIT, C. T. 1960. On competition. Versl. Landb. Onderz., 66, $1-82$. 